\title{
Introducción de metodologías de Aprendizaje Basado en Problemas mediante tecnologías multimedia
}

\author{
Juan M. Orduña, Rafael Sebastian, and Ricardo Olanda \\ Departamento de Informática, Universidad de Valencia, Spain \\ \{juan.orduna, rafael.sebastian,ricardo.olanda\}@uv.es
}

Resumen. La propuesta de trabajar por competencias en lugar de hacerlo como se hacía tradicionalmente, por objetivos, ha hecho proliferar las propuestas y alternativas metodológicas para favorecer un cambio en la Educación Superior. Una de estas metodologías es el aprendizaje basado en problemas (ABP). El ABP es una metodología de aprendizaje en la cual el punto de partida es un problema construido por el profesor que permite al estudiante identificar necesidades para comprender mejor ese problema, identificar principios que sustentan el conocimiento y cumplir objetivos de aprendizaje relacionados con cada porción del contenido de la materia. En el caso de las tecnologías de la información y las comunicaciones es especialmente relevante, ya que permite desarrollar simultáneamente conocimientos teóricos y estrategias para resolver problemas prácticos en pequeños grupos, similares a los que se encuentran en la práctica profesional. La organización en grupos de trabajo permite además la creación de contenido de caracter longitudinal y transversal al grupo. El contenido transversal permite el uso de técnicas de aprendizaje colaborativo, que se pueden combinar con tecnologías multimedia en la web 2.0 para facilitar su difusión entre grupos y a la comunidad en general.

En este artículo se describe la experiencia docente en la asignatura de Arquitectura de Redes de Computadores impartida en los grados de Ingeniería Informática, Ingeniería Electrónica e Ingeniería Electrónica de Telecomunicaciones de la Universitat de Valencia. En ella, los alumnos de los tres grados han sido expuestos a la metodología de ABP y de aprendizaje colaborativo basado en tecnologías multimedia. Los contenidos de los grados se han adaptado adecuadamente de forma transversal permitiendo desarrollar los contenidos teórico prácticos. Los resultados de esta experiencia docente ha sido publicados en la web, así como los materiales generados por los grupos de alumnos. Todo el proceso ha sido supervisado por los profesores de la asignatura, obteniendo una retroalimentación continua acerca de su trabajo por medio de comentarios y evaluaciones realizados tanto por parte de los profesores como por alumnos externos al grupo de trabajo.

The introduction of skills-based learning instead of the more traditional goal-based learning has given rise to novel teaching proposals and methodological alterna tives that favor a change in the context of the Euro- pean 
Higher Education Area. One of these methods is problem/project-based learning $(\mathrm{PBL})$. PBL is a learning methodology in which the starting point is a problem constructed by the teacher, which allows students to identify needs to better understand the problem/situation, identify principles underpinning knowledge and meet learning objectives related to each content part. In the case of Information and Communications technologies it is especially relevant, since it allows the concurrent development of theoretical knowledge and strategies for solving problems in small groups, similar to those found in practice. This methodolgy can also be combined with the collaborative learning and the use of multimedia and web technologies for presenting the work done. It improves both the degree of motivation of the students and the degree of disseminations of the works. In this paper, we describe the teaching experience in the course of Computer Networks Architecture taught in undergraduate degrees of Computer Engineering, Telematics Engineering and Telecommunications Electronic Engineering from the University of Valencia. The students have been divided into working groups, and they have developed digital objects of learning that have been freely disseminated through Internet. This methodology has provided the students not only with collaborative learning within each group, but also with inter-group collaborative learning. Each group obtained feedback from the comments and evaluations of other students and the course teachers.

Keywords: Aprendizaje basado en problemas, aprendizaje colaborativo, tecnologías multimedia

Keywords: Problem-Based Learning, collaborative learning, multimedia technology

\section{Motivación}

Las metodologías docentes tradicionales basadas en la transmisión de conocimiento del docente al alumno no benefician el desarrollo de las competencias necesarias para el trabajo profesional de ingenieros en tecnologías de la información y las telecomunicaciones (TIC). Este tipo de trabajo suele desarrollarse a través de proyectos que presentan retos técnicos a equipos de ingenieros. Además, debido a la constante evolución de la tecnología, es necesario que los ingenieros TIC sean capaces de aprender de forma autónoma, y de aplicar conocimientos teóricos a la práctica.

Una técnica docente que se adapta a estos requisitos es el aprendizaje basado en problemas (ABP). Este método promueve en el alumno la responsabilidad de su propio aprendizaje, en lugar de que este sea un mero recipiente pasivo de información. Se fomenta la motivación del alumno para que aprenda de forma autónoma, y el profesor pasa de tener un rol de experto, a un rol de facilitador o tutor del aprendizaje. Con ello se consigue desarrollar habilidades para la evaluación crítica y la adquisición de nuevos conocimientos con un compromiso de aprendizaje de por vida. 
El desarrollo de problemas de tamaño medio o grande facilita la posibilidad de llevarlos a cabo en grupos reducidos y a lo largo todo el curso. El ABP permite el planteamiento de problemas que puedan ser evaluados incrementalmente mediante documentos similares a los utilizados en proyectos reales (informes), sobre los que el profesor pueda ofrecer feedback que ayude a los alumnos a reflexionar y profundizar. En este marco, se consigue fomentar habilidades para las relaciones interpersonales y trabajo colaborativo. Por otra parte, el trabajo colaborativo se puede definir como actividades de aprendizaje expresamente diseñadas para grupos pequeños de alumnos que interactúan entre sí [2]. Las propuestas que han evaluado la efectividad del trabajo colaborativo muestran que los resultados son positivos para el proceso de aprendizaje $[5,10]$. Son varias las técnicas existentes para aplicar esta metodología colaborativa. Algunos autores han hecho una comparativa de algunas de estas técnicas, como por ejemplo Hausmann [9], que se centra en 3 técnicas distintas: explicación directa, co -construcción y explicación por parte de otra persona. En base al estudio comparativo, se concluye que la explicación por parte de otra persona obtiene los mejores resultados en cuanto a cantidad de información aprendida, mientras que la co -construcción obtiene mejores resultados para la resolución de problemas.

En este artículo se describe la experiencia de unir la implantación y desarrollo de la metodología ABP con el aprendizaje colaborativo a través de la elaboración de una tarea longitudinal correspondiente a un proyecto, junto a tareas colaborativas transversales en forma de objetos digitales de aprendizaje. La división del trabajo en diferentes grupos se ha realizado siguiendo la técnica del puzzle de Aronson [1].

La metodología se ha aplicado a la asignatura de Arquitectura de Redes de Computadores, impartida en la titulaciones de Grado de Ingeniería Electronica de las Telecomunicaciones, Grado de Informática y Grado de Telemática de la Universidad de Valencia. ésta metodología ha permitido trabajar competencias específicas importantes tales como el trabajo en equipo o la comunicación oral. Los resultados obtenidos en las diferentes partes evaluables de la asignatura han demostrado una mayor implicación de los alumnos en los trabajos en grupo, si se compara con los resultados de carácter individual. Por otra parte, los objetos digitales de aprendizaje han permitido a los alumnos, además de adquirir los conocimientos propios derivados de su desarrollo, hacer uso de ellos como elementos constructivos básicos para la elaboración de unproyecto complejo que los englobe, sirviéndoles a su vez como recursos docentes disponibles en todo momento. Estos objetos estarán disponibles de forma libre para el resto de compañeros y para el público en general.

El resto de este artículo se divide en las siguientes secciones: en la sección 2 se revisa la metodologías del ABP y las diferentes técnicas de aprendizaje colaborativo. En la sección 3 de detalla el contexto y la realización de la experiencia. En la sección 4 se aportan los resultados extraídos de esta experiencia. Por último, en la sección 5 se presentan las conclusiones. 


\section{Estado del arte}

\subsection{Aprendizaje Basado en Problemas}

El ABP es una metodología docente basada en el estudiante como protagonista de su propio aprendizaje. Es una estrategia educativa que hace descansar el proceso de aprendizaje del alumno sobre un problema construido por el profesor, similar a uno de la vida real, que sirve como vehículo, ya sea para la adquisición de un cuerpo integrado de conocimientos sobre la materia, o para la adquisición de competencias en la resolución de problemas prácticos [3, 13, 12]. En el ABP, los estudiantes se reunen en torno a un problema antes de recibir instrucción alguna sobre cómo resolverlo. El ABP representa toda una revolución respecto de los planes de estudios tradicionales donde los roles de profesor y alumno cambian. El papel del profesor en un aula de ABP es similar al de un guía o entrenador, es decir, el de facilitador, en lugar del poseedor de todo el conocimiento. Por ello, este enfoque requiere problemas o casos especialmente diseñados para motivar el aprendizaje de los aspectos más relevantes de la materia o disciplina de estudio. La colaboración y el aprendizaje cooperativo es esencial para el éxito del ABP [11]. El aprendizaje cooperativo de los estudiantes se realiza en pequeños grupos de estudiantes, y puede ser la parte más gratificante y productiva de su aprendizaje.

\subsection{Aprendizaje Colaborativo Basado en Problemas}

Con la aparición de las nuevas tecnologías se ha abierto un amplio abanico de técnicas para el diseño de tareas de trabajo colaborativo.

En el contexto de la docencia en Ingenierías existen también varios los trabajos que se han desarrollado para tratar el trabajo colaborativo. Uno de ellos se ha centrado en estudiar el efecto que tiene incorporar las redes sociales (en especial facebook) dentro del proceso de aprendizaje colaborativo [8]. Los resultados del trabajo muestran que se produce una mejora en la comunicación entre profesor y alumno. En otros trabajos se ha estudiado el aprendizaje colaborativo en Ingeniería Informática a través de herramientas Web 2.0, utilizando blogs, wikis y la plataforma virtual de la universidad Vaquerizo [14]. Los resultados muestran que la Web 2.0 es un medio de participación, comunicación y trabajo en equipo efectivo, pero el problema surge a la hora de evaluar a cada individuo del grupo por separado. Son varios los trabajos que mencionan YouTube como una herramienta Web 2.0 que se puede utilizar para aplicar el trabajo colaborativo $[6,7]$ de forma que permita la divulgación del contenido de aprendizaje desarrollado.

\section{Aprendizaje Basado en Problemas apoyado en Objetos Digitales de Aprendizaje}

Para aplicar la metodología de ABP en la asignatura de ARC, los alumnos organizados en grupos, deben llevar a cabo el diseño de una red de computadores que satisfaga las necesidades de comunicaciones de una empresa de gran tamaño. 
En su desarrollo, se realiza un estudio detallado de cada una de las etapas de desarrollo e implantación de una red de computadores: definición del problema, diseño físico, diseño lógico e implantación de aplicaciones de red.

Para ayudar a los grupos en el desarrollo del proyecto, los propios alumnos elaborarán un conjunto de objetos digitales de aprendizaje transversales que profundizarán en cada uno de los puntos teóricos de diseño a tratar. Además el profesor les entregará un cuaderno de trabajo, donde se recogen los requisitos mínimos que debe incluir un proyecto, las diferentes fases del mismo, los roles que pueden desempeñar los alumnos, un calendario con las fechas límite para cada una de las fases del proyecto y los criterios de evaluación que se van a utilizar para valorar el mismo. La mecánica empleada para esta metodología es la siguiente:

- El profesor definirá las características del proyecto y de los objetos digitales de aprendizaje transversales a realizar, y proporcionará a los alumnos un cuaderno de trabajo para ayudarles a organizarse en el desarrollo del mismo.

- Para la constitución de los grupos se utilizará la técnica del puzzle de Aronson. El proceso de creación de grupos se detalla en la sección 3.1.

- Cada grupo de expertos elaborará el objeto de aprendizaje que le corresponde según cada fase del proyecto y lo envirá al profesor en formato de video presentaciones 3.2 .

- Todos los videos elaborados se colgarán en un canal de YouTube público de la asignatura. Los videos estarán disponibles antes de que los alumnos comiencen la realización del proyecto de diseño.

- Mensualmente los alumnos, dentro de sus grupos nodriza, resolverán el proyecto de diseño para cada uno de los niveles (físico, lógico y aplicación), elaborando un documento escrito para cada uno de ellos.

- Cada grupo nodriza realizará una exposición oral de la solución propuesta para cada nivel del proyecto delante del profesor y de los otros grupos de compañeros.

- Cada grupo nodriza presentará su trabajo definitivo a través de un documento escrito y junto a un video describiendo el mismo o un poster, en el cual se resumirán las ideas fundamentales del proyecto.

- La evaluación de la resolución final del proyecto será evaluada por el profesor de la asignatura, por otros grupos de alumnos y por profesores externos a la asignatura, obteniendo una valoración que se complementará con la valoración del documento escrito del proyecto realizada por el profesor de la asignatura.

El desarrollo tanto de los objetos digitales de aprendizaje como del proyecto por parte de los alumnos debe realizarse de forma mixta, fuera del aula (la elaboración de los objetos de aprendizaje, el desarrollo del documento escrito y la preparación de las exposiciones orales del proyecto), para lo cual se emplearán las horas de trabajo fuera del aula especificadas en la guía docente, y en la propia aula (exposiciones orales y evaluación por compañeros), dentro de las horas de problemas contempladas en la planificación de la asignatura. De esta forma, la carga de trabajo del alumno se adecuará a la especificada en la guía docente. 


\subsection{Formación de los grupos}

Como se ha comentado, se va a emplear la técnica del puzzle de Aronson para constituir los diferentes grupos que han de desarrollar los objetos digitales de aprendizaje y el proyecto de diseño de una red de computadores. El objetivo es que en cada grupo de desarrollo del proyecto de diseño exista al menos un experto en cada uno de los niveles de desarrollo del mismo. Los alumnos formarán grupos de 4 o 5 integrantes para la realización del proyecto de diseño de la red de computadores. Estos grupos se denominan grupos nodriza. Los alumnos han de desarrollar tres objetos digitales de aprendizaje asociados a los diferentes niveles de diseño de una red de computadores: físico, lógico y de aplicación. Para ello se crean diferentes grupos de expertos que están compuestos por uno o varios alumnos de cada uno de los grupos nodriza. Se ha de asegurar que cada grupo nodriza tenga al menos un miembro en cada uno de esos grupos de expertos. Es responsabilidad de los expertos, una vez hayan elaborado el objeto digital de aprendizaje correspondiente, explicar y compartir los conocimientos adquiridos dentro del entorno del grupo de expertos al resto de miembros del grupo nodriza, para que todos adquieran las competencias adecuadas en cada uno de los niveles de diseño.

\subsection{Objeto digital de aprendizaje transversal}

Los objetos digitales de aprendizaje que van a realizar los alumnos son videos multimedia. La base de estos videos multimedia está formada por transparencias con una plantilla predeterminada que son explicadas mediante la grabación de la voz en off de uno de los miembros del grupo. El vídeo se deberá centrar de forma teórico-práctica en la explicación de uno solo de los niveles de diseño de la red de computadores. Para su publicitación se usa una página web dentro del canal de videos YouTube púbblico. Adicionalmente a la realización de estos videos multimedia, los alumnos pueden elaborar de forma opcional un video multimedia que incluya la presentación final de todo su proyecto.

\section{Resultados}

La aplicación de la metodología de ABP en la asignatura de ARC se llevo a cabo a lo largo de cinco sesiones presenciales de dos horas en los espacios dedicados a seminarios/problemas. Adicionalmente, los estudiantes reportaron un total de entre seis a diez horas de dedicación por miembro del grupo para completar cada una de las fases del proyecto. Estas horas incluían tanto reuniones de todo el grupo $(30 \%)$ como trabajo individual $(70 \%)$. Todos los miembros de cada grupo fueron al menos una vez expertos de su grupo y realizaron una presentación oral al resto de la clase, lo cual ayudó a mejorar las competencias de comunicación oral y presentaciones frente a un público.

La figura 1 muestra, a modo de ejemplo, las puntuaciones globales obtenidas por los cinco proyectos realizados en el grado de Ingeniería Informática. Las 
puntuaciones reflejan la calificación media de todas las sesiones destinadas a presentar las distintas partes del proyecto (especificaciones de usuario, diseño físico, diseño lógico, etc.) obtenidas por cada grupo de alumnos que realizaba un proyecto concreto. Para cada grupo hay 2 valores: la puntuación media otorgada por el profesor y la puntuación media otorgada por el resto de alumnos de la clase. La puntuación del resto de alumnos se realizó también de forma grupal; los miembros de cada grupo consensuaban una nota para el resto de grupos en cada sesión, en base a las presentaciones realizadas por estos y en base a las respuestas y discusiones surgidas después de cada presentación.

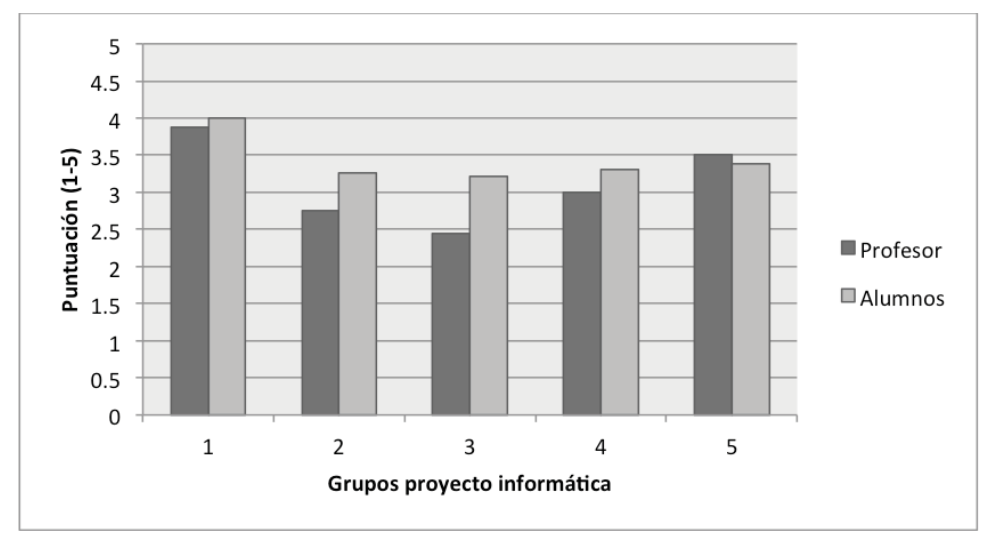

Fig. 1. Puntuaciones obtenidas en cinco proyectos en el grado de informática.

Los resultados de la figura 1 muestran que tanto para el grupo 1 como para el grupo 5 las puntuaciones otorgadas por el profesor y por el resto de los alumnos son muy parecidas, sin diferencias significativas. Sin embargo, para los grupos 2 , $3, y 4$ se observa que las puntuaciones otorgadas por el profesor son significativamente inferiores a las otorgadas por el resto de alumnos. Es de destacar que estas diferencias se deben en su gran mayoría a que los grupos presentaban trabajos donde algunos de los aspectos evaluados (p. ej. claridad/precisión/corrección de las respuestas) eran claramente deficientes, bien por ausencia o bien por incorrección de los contenidos. Si bien estas deficiencias quedaban explicitadas durante la discusión, los alumnos tendían a no reducir la nota de sus compañeros.

Por otro lado, para evaluar el uso de los objetos digitales de aprendizaje elaborados, se ha utilizado como métrica el número de visualizaciones que se han realizado de cada uno ellos. Estos vídeos se expusieron en una página de www.youtube.es/ARCuv2013. Además, también hemos analizado los resultados de la encuesta anónima acerca del uso de esta metodología rellenada por los alumnos.

En la figura 2 se muestra el número de visitas que ha recibido cada uno de los videos multimedia realizados por los alumnos. En esa figura se han agrupado por columnas los diferentes grupos de expertos que han realizado el mismo tipo de 
video multimedia. El apartado de diseño del nivel físico (D.F.), se ha subdivido en tres, haciendo referencia al cableado de cobre, al de fibra y a los diferentes dispositivos de red.

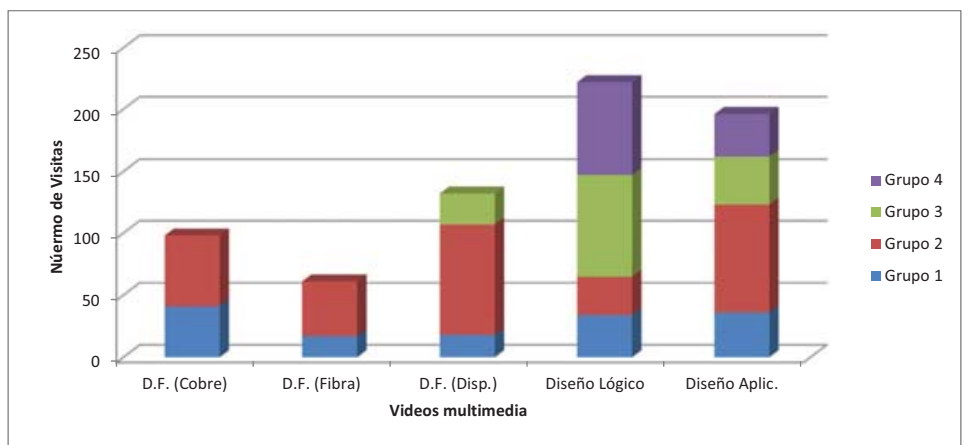

Fig. 2. Número de visitas recibido por cada uno de los videos multimedia

En la figura se observa que, para el nivel de diseño físico, a excepción de los videos multimedia referidos al cableado de fibra óptica (61 visitas en total para los dos videos), el número de visitas es superior al número de alumnos (que es $65)$, por lo que en media, podríamos decir que todos los alumnos han visto al menos una vez, un video multimedia de cada una de estas categorías.

El número global de visitas para el diseño físico sería de 291, lo que implica una media superior a 4 visitas por alumno a algún video de esta categoría. Para el diseño lógico el número total de visitas es de 222 y para el de aplicación de 196, lo cual supone una media de visitas por alumno superior a 3 en ambos casos. En cuanto a cada video particular, el número de visitas varía entre 17 y 89, lo que podría considerarse como un índice de calidad del mismo, ya que si le resulta útil al alumno, lo volverá a visualizar.

Finalmente, se realizó una encuesta anónima que constaba de las siguientes preguntas:

- He visualizado los videos de los diferentes niveles de desarrollo del proyecto.

- La visualización de estos videos para la realización del proyecto me ha resultado de gran utilidad.

- La realización del video me ha ayudado a comprender mejor ese nivel de desarrollo del proyecto.

- La técnica del puzzle de Aronson me ha parecido interesante.

Las respuestas posibles a estas preguntas son, para la primera pregunta, sí o no. Para el resto de preguntas, las respuestas abarcan una escala de Likert de 5 puntos, en función de lo de acuerdo que esté el alumno con la afirmación ( $1=$ nada, 5 = totalmente de acuerdo).

Todos los alumnos respondieron afirmativamente a la primera pregunta, lo que supone que han hecho uso del material que se ha realizado. Los resultados 
de las restantes preguntas (numeradas de 2 a 4) se muestran en la figura 3, donde se ha calculado la media de las respuestas de los alumnos (siguiendo las recomendaciones de trabajos previos sobre el análisis de las Likert [4]). En esa figura se puede observar que, en general, los alumnos ven útil la realización de estos videos. Cabe destacar que los alumnos indican que han aprendido más realizando el video (media en torno a 4) que viéndolo (media en torno a 3.7). La técnica del puzzle de Aronson, también la ven interesante, a pesar de obtener una valoración más baja (en torno al 3.3).

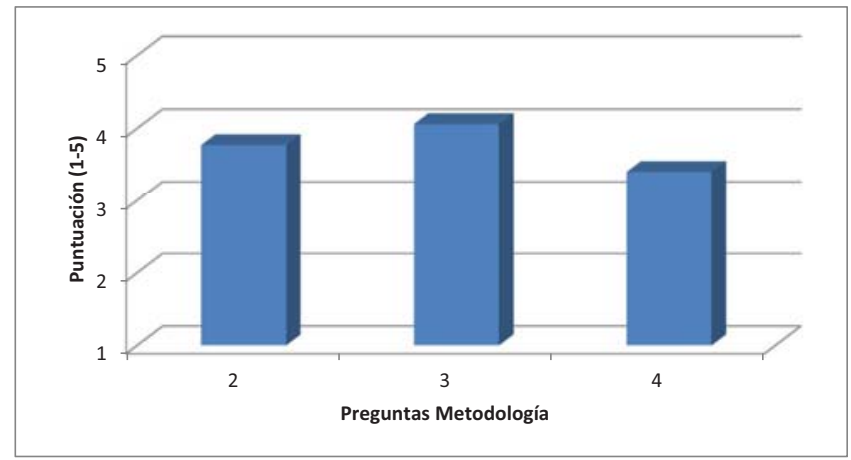

Fig. 3. Encuestas anónimas sobre la metodología docente

\section{Conclusiones}

En este trabajo hemos mostrado una experiencia de aplicación de aprendizaje basado en problemas unida a un aprendizaje colaborativo mediante la elaboración de objetos digitales de aprendizaje. Los alumnos han realizado diversos videos multimedia que se han convertido en objetos digitales de aprendizaje disponibles en un canal de YouTube. El acceso a estos videos es libre tanto para los alumnos de la asignatura como para el público en general. La aplicación de la metodología $\mathrm{ABP}$ ha demostrado ser beneficiosa para los alumnos, ya que ha permitido trabajar competencias específicas importantes tales como el trabajo en equipo o la comunicación oral. Los resultados obtenidos en las diferentes partes evaluables de la asignatura han demostrado una mayor implicación de los alumnos en los trabajos en grupo, si se compara con los resultados de carácter individual.

Por otra parte, las estadísticas de visitas reflejan que se ha hecho uso de los objetos digitales (vídeos) desarrollados, ayudando a los alumnos en la elaboración de su proyecto de diseño de una red de computadores. El uso de estos videos se hace patente a la hora de evaluar los proyectos, donde se observa una mejora de la calidad de los mismos respecto a cursos anteriores. Finalmente, los propios alumnos manifiestan que consideran útil esta metodología, ya que les 
ha permitido comprender mejor los conceptos necesarios para el desarrollo del proyecto.

\section{References}

1. Aronson, E., Patnoe, S.: The jigsaw classroom: building cooperation in the classroom. Longman (1997), http://books.google.es/books?id=L0KfAAAAMAAJ

2. Barkley, E.F.: Técnicas de aprendizaje colaborativo: manual para el profesorado universitario. Ediciones Morata (2007)

3. Barrows, H., Tamblyn, R.: Problem-based Learning: An Approach to Medical Education. Springer Publishing Company (1980)

4. Blaikie, N.: Analyzing quantitative data: From description to explanation. Sage Publications (2003)

5. Dillenbourg, P., Baker, M., Blaye, A., O'Malley, C.: The evolution of research on collaborative learning. In: (Eds), E.S..P.R. (ed.) Learning in Humans and Machine: Towards an interdisciplinary learning science., pp. 189-211 (1995)

6. Esteve, F.: Bolonia y las TIC: de la docencia 1.0 al aprendizaje 2.0. La Cuestión Universitaria 5 (2009)

7. García, F., Portillo, J., Romo, J., Benito, M.: Nativos digitales y modelos de aprendizaje. In: Benito, M., Romo, J., Portillo, J. (eds.) SPDECE. CEUR Workshop Proceedings, vol. 318. CEUR-WS.org (2007)

8. Garrigós, I., Mazón, J.N., Saquete, E., Puchol, M.: La influencia de las redes sociales en el aprendizaje colaborativo. In: XVI Jornadas de Enseñanza Universitaria de la Informática. pp. 531-534. JENUI (2010)

9. Hausmann, R., Chi, M.T., Roy, M.: Learning from collaborative problem solving: An analysis of three hypothesized mechanisms. 26nd annual conference of the Cognitive Science society pp. 547-552 (2004)

10. Hennessy, S., Murphy, P.: The potential for collaborative problem solving in design and technology. International Journal of Technology and Design Education pp. 1$36(1999)$

11. Molina, J., Garcia, A., Pedraz, A., Anton, M.: Aprendizaje basado en problemas: una alternativa al metodo tradicional. Revista de la Red Estatal de Docencia Universitaria 3(2), 79-85 (2003)

12. Morales, P., Landa, V.: Aprendizaje basado en problemas. Theoria 13, 145-157 (2004)

13. Savery, J.: Overview of problem-based learning: Definitions and distinctions. The Interdisciplinay Journal of problem-Based Learning 1, 9-29 (2006)

14. Vaquerizo, B., Renedo, E., Valero, M.: Aprendizaje colaborativo en grupo: Herramientas web 2.0. In: XVI Jornadas de Enseñanza Universitaria de la Informática. pp. 447-450. JENUI (2009) 